\title{
Aspectos clínicos, biológicos, histopatológicos e tratamentos propostos para a mucosite oral induzida por radioterapia: revisão da literatura
}

\author{
Clinical, biological, histological features and treatment of oral mucositis induced \\ by radiation therapy: a literature review
}

\author{
Paulo Rogério Ferreti Bonan,'Márcio Ajudarte Lopes, ${ }^{2}$ Fábio de Abreu Alves, ${ }^{3}$ Oslei Paes de Almeida, ${ }^{2}$
}

\section{Resumo}

A mucosite oral é um dos principais efeitos colaterais agudos induzidos pelo tratamento radioterápico em cabeça e pescoço, surgindo a partir da segunda semana de tratamento e é caracterizada por ardência bucal até sintomatologia dolorosa intensa exigindo em algumas situaçōes a interrupção do tratamento radioterápico. O objetivo desse trabalho foi rever os principais trabalhos publicados que discutem as características clínicas, histopatológicas e tratamentos propostos para a mucosite oral induzida por radioterapia. Poucos estudos descreveram os aspectos histopatológicos da mucosite oral e apenas recentemente os mecanismos biológicos começaram a ser desvendados. Embora diversas modalidades terapêuticas sejam indicadas para a prevenção e tratamento da mucosite oral, não existe consenso sobre o tratamento mais indicado. A melhor compreensão dos mecanismos biológicos e da histopatologia provavelmente contribuirá para a indicação de terapias eficazes na prevenção e manejo da mucosite oral.

Palavras-chave: Radioterapia; Mucosite; Neoplasias bucais; Efeitos adversos.

\section{Abstract}

The oral mucositis is a main side effect of radiotherapy on head and neck, initiating two weeks after the beginning of the treatment. It is characterized by sensation of local burning to intense pain, leading in several cases, to the interruption of the treatment. The purpose of this work is to review the main published studies that discusse the clinical, biological and histopathological features of oral mucositis induced by radiation therapy and to describe the main approches recommended to prevent or to treat it. Although the clinical features of mucositis are intensively described in the literature, few studies address the histopatological alterations in oral mucositis and only recently, its biological processes have been investigated. The biological mechanisms involved in the radiation tissue damage have been only recently discussed and there is no consensus among treatment modalities. Yet, the progressive knowledge in the histopathology and biological characteristics of oral mucositis probably will lead to more effective in prevention and control strategies.

Key words: Radiotherapy; Mucositis; Mouth neoplasms; Adverse effects.

\footnotetext{
${ }^{1}$ Professor Doutor, Sub-Área de Estomatologia, Departamento de Odontologia, Universidade Estadual de Montes Claros e Faculdades Unidas do Norte de Minas, MG.

2 Professor Doutor, Departamento de Diagnóstico Oral, Área de Patologia, Faculdade de Odontologia de Piracicaba, Universidade Estadual de Campinas, UNICAMP, SP

${ }^{3}$ Professor Doutor, Departamento de Estomatologia do Hospital do Câncer AC Camargo, Faculdade de Odontologia, UNINCOR, SP. Endereço para correspondência: Paulo Rogério Ferreti Bonan. Av.Corinto Crisóstomo Freire,600/305H, Morada do Parque Montes Claros, MG CEP: 39401-365. E-mail: pbonan@yahoo.com
} 


\section{INTRODUÇÃO}

A radioterapia, exclusiva ou adjuvante à cirurgia, é efetiva no tratamento e controle dos carcinomas espinocelulares em cabeça e pescoço. ${ }^{1-3}$ As opções de tratamento radioterápico se dividem em duas modalidades: teleradioterapia (radioterapia com fonte a distância) e a braquiterapia (radioterapia por meio do implante, através de aplicadores intra-luminais ou endocavitários e contato direto de radioisótopos). A teleradioterapia pode ser feita com equipamentos de cobaltoterapia ou aceleradores lineares onde técnicas de planejamento convencionais, tridimensionais ou conformacionais são empregadas. ${ }^{1-7}$ Embora a teleterapia seja muito utilizada, seus efeitos colaterais afetam particularmente a cavidade bucal. ${ }^{1,2,5,8,9}$ Alteraçōes nas regiôes irradiadas afetando mucosas, ossos, glândulas salivares, dentes, estruturas vasculares, nervosas e musculares são freqüentemente relatadas. ${ }^{8,10-13}$

Xerostomia, trismo, cárie por radiação, candidose, osteorradionecrose, hiperpigmentaçôes em mucosa e mucosite são os principais efeitos colaterais da radioterapia em cavidade bucal., ${ }^{2,5,10-15}$ Pacientes irradiados em região de cabeça e pescoço que responderam a um questionário sobre alteraçōes orais e qualidade de vida, relataram que a boca seca $(91,8 \%)$, alteraçōes na gustação $(75,4 \%)$, disfagia $(63.1 \%)$ e dor $(58,4 \%)$ que interferia diretamente nas atividades diárias em $30,8 \%$ dos casos foram os principais sintomas encontrados em decorrência do tratamento radioterápico.?

A mucosite oral é o efeito colateral agudo mais importante da radioterapia em cavidade bucal, ${ }^{16-24}$ por dificultar a deglutição de alimentos sólidos e às vezes líquidos, limita a fala e a mastigação, além de expor o paciente a infecções por microorganismos oportunistas, resultando na diminuição da qualidade de vida do paciente irradiado. ${ }^{3,16,18,25}$ Além disso, a mucosite oral grave pode exigir interrupção parcial ou completa da radioterapia antes do regime planejado ser completado, aumentando o risco de proliferação das células tumorais e dificultando o controle do câncer. ${ }^{24}$ Uso do álcool e tabaco, associação com a quimioterapia, hiperfracionamento, infecções fúngicas e má higienização bucal podem aumentar a incidência ou agravar a mucosite. . $^{1826}$

O objetivo desse trabalho foi rever os principais estudos publicados (Medline de 1981 a 2005) que discutem as características clínicas, histopatológicas e tratamentos propostos para a mucosite oral induzida por radioterapia.

\section{PrevalênCIa e Características ClínICAS}

A mucosite oral induzida por radioterapia acomete praticamente todos os pacientes submetidos à radiação tumoricida em campos cérvico-faciais. ${ }^{16}$ Trotti et al, ${ }^{26}$ em um estudo metanalítico, relataram após análise de diversos estudos sobre mucosite oral, que $97 \%$ de 2875 pacientes desenvolveram mucosite devido à radioterapia convencional. A associação entre radioterapia e quimioterapia, resultou em mucosite oral em $89 \%$ de 1505 pacientes irradiados, comparados apenas a $22 \%$ de 318 pacientes que desenvolveram mucosite oral pela realização de quimioterapia exclusiva. A insuficiência de dados e sua heterogenicidade impediram a análise da severidade da mucosite e a maior incidência de mucosite esteve diretamente associada com o uso de doses hiperfracionadas. Handschel et al, ${ }^{27}$ que acompanharam 13 pacientes irradiados em cabeça e pescoço, reportaram que todos os pacientes desenvolveram mucosite oral a partir da segunda semana do início da radioterapia apresentando ardência e eritema na faixa de 3000 cGy até ulceraçóes confluentes e extensas por volta de 6000 cGy.

A incidência e intensidade da mucosite dependem do tipo de radiação ionizante (elétrons ou $\mathrm{Co}^{60}$ ) empregada, taxa de distribuição, dose total empregada, conjugação com agentes quimioterápicos como Metotrexato, 5-Fluorouracil, Bleomicina e Cisplatina, resposta individual a terapia, fatores como o uso do tabaco e do álcool e níveis de EGF (fator de crescimento epitelial) e PAF (fator de ativação plaquetária) presentes na saliva. ${ }^{18,26,28-33}$ Pacientes submetidos à radioterapia com hiperfracionamento de dose, apresentaram maiores incidências de mucosite (100\%) do que os pacientes que fizeram radioterapia convencional (97\%), associada à quimioterapia $(90 \%)$ ou que fizeram quimioterapia exclusiva (22\%). ${ }^{26,33} \mathrm{Um}$ estudo realizado por Denham et al ${ }^{30} \mathrm{com} 191$ pacientes irradiados em cabeça e pescoço, evidenciou a importância da resposta individual à terapia na manifestação da mucosite oral ulcerativa confluente bem como o papel sinérgico do tabagismo, consumo de álcool, infecçõos dentais, status nutricional e má higiene oral. Níveis elevados de PAF na saliva de pacientes em tratamento radioterápico em cabeça e pescoço estão associados a episódios mais severos de mucosite oral, devido à indução a inflamação existente na mucosite oral. ${ }^{28}$ Em contrapartida níveis baixos de EGF salivar estão diretamente associados à intensidade da mucosite oral, resultando na deficiência na reparação da mucosa irradiada. ${ }^{32}$

Outro fator agravante para a mucosite oral é a susceptibilidade à infecção por microorganismos normalmente presentes na cavidade bucal que são oportunistas e invadem os tecidos lesados. Leveduras 
do gênero Candida aumentam a colonização durante a radioterapia. ${ }^{29,34,35}$ Embora se tenha aventado a hipótese de que bactérias Gram negativas tenham um papel crucial no desenvolvimento da mucosite oral, dois estudos que procuraram erradicar seletivamente essas bactérias e Candida não conseguiram atenuar a mucosite induzida por radioterapia. ${ }^{36,37}$

Clinicamente, duas semanas após o início da radioterapia em cabeça e pescoço, a mucosa oral se torna esbranquiçada, seguindo-se eritema, e por volta de 2500 cGy há perda de espessura da mucosa e em poucos dias surgem ulcerações recobertas por fibrina ${ }^{29}$ (Figuras 1 e 2). A diferenciação clínica entre a mucosite intensa e processos infecciosos como a candidose pseudomembranosa pode ser difícil já que podem se apresentar sobrepostos $^{38}$ (Figura 3). A mucosa faringeana parece ser mais sensível à radiação sendo o local onde a dor durante a alimentação se inicia, induzindo o paciente a recorrer a dietas com alimentos leves. ${ }^{17,39} \mathrm{O}$ quadro sintomatológico resultante da mucosite varia da queixa de ardência bucal a dor intensa e sangramento espontâneo que podem impedir a alimentação e que, eventualmente, conduzem o paciente a caquexia e a nutrição parenteral ou através de sondas nasogástricas. ${ }^{16,26}$ Aproximadamente $11 \%$ dos casos de interrupção e modificação do regime de tratamento radioterápico ocorrem devido ao desenvolvimento de mucosite oral intensa, sendo necessário o replanejamento do tratamento e em algumas situações clínicas, a internação do paciente. $^{2}$ Para a maioria dos pacientes, 10 a 14 dias são suficientes para o reparo do tecido lesado pela radiação, mas para pacientes que têm reparo deficiente ou que recebem doses acima de 6.500 cGy, esse período varia de 14 a 21 dias. . $3,30^{3}$

A mensuração da intensidade da mucosite oral pode ser feita através de índices descritos pela Organização Mundial da Saúde (OMS); $;{ }^{27}$ pelo método de Hickey; ${ }^{16}$

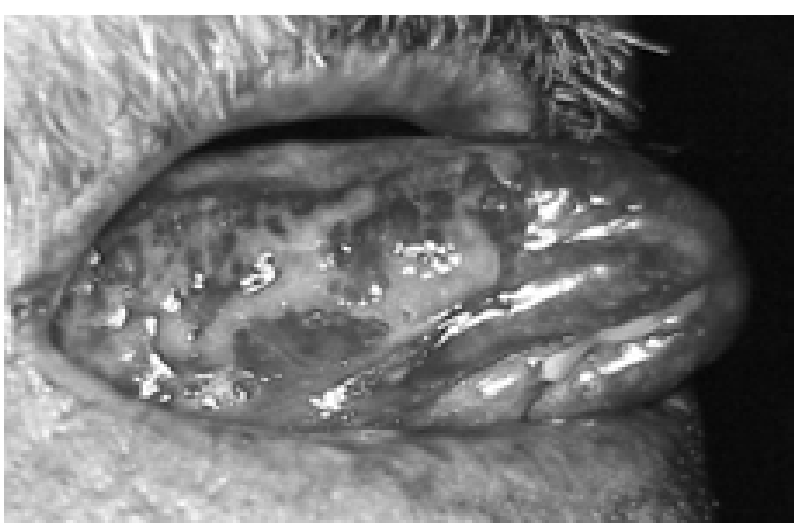

Figura 1. Ulcerações confluentes recobertas por membrana fibrinopurulenta em língua e comissura de paciente em curso radioterápico em cabeça e pescoço.

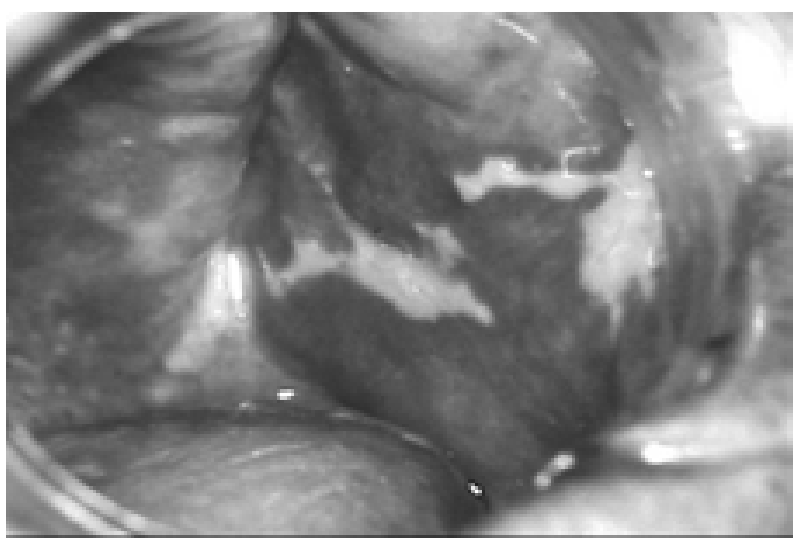

Figura 2. Eritema generalizado e ulcerações recobertas por membrana fibrinopurulenta em mucosa bucal em paciente durante radioterapia em cabeça e pescoço.

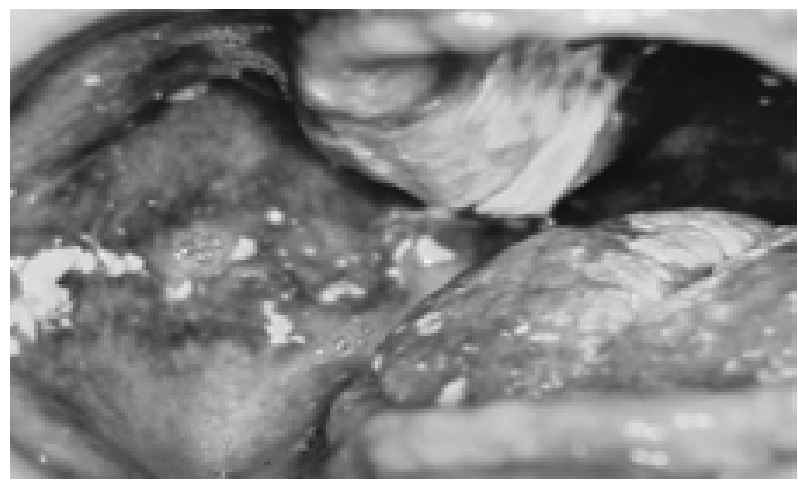

Figura 3. Candidose pseudomembranosa sobreposta em mucosite induzida por radioterapia.

método de Van Der Schuren; ${ }^{16}$ método de Spijkervet; ${ }^{16-17}$ classificação RTOG/EORTC (Radiation Therapy Oncology Group/ European Organization for Research and Treatment of Cancer) ${ }^{16}$ e pela classificação proposta por Stokman et al ${ }^{41}$ para a avaliação do número de células bem diferenciadas viáveis esfoliadas da mucosa bucal irradiada. Os estudos mais recentes sobre mucosite oral utilizaram principalmente a classificação proposta pela OMS, que faz a graduação da mucosite em 4 níveis (I ardência, eritema; II - eritema, úlceras, dieta sólida; III - confluência de úlceras, dieta líquida; IV - alimentação via oral não é possível, dieta líquida). ${ }^{26}$ Mucosite em graus III e IV (OMS) após a metade do tratamento radioterápico em doses tumoricidas são freqüentes. ${ }^{27}$ Cerca da metade dos pacientes que recebem radioterapia fracionada (56\%) desenvolvem mucosite graus III e IV em comparação com $34 \%$ dos pacientes que recebem tratamento radioterápico convencional e associação com agentes quimioterápicos, tabagismo e fumo são fatores predisponentes. ${ }^{18,26}$ 


\section{Patogenia e Características histopatológicas}

A mucosite oral como processo biológico tem sido apenas recentemente estudada, e se tem sugerido mecanismos envolvendo a mucosa oral, citocinas próinflamatórias e fatores locais como a saliva e a microbiota em sua patogênese. ${ }^{42,43} \mathrm{~A}$ mucosite induzida por radioterapia e quimioterapia pode ser dividida em quatro fases distintas: vascular, epitelial, ulcerativa e de reparo. ${ }^{42,43}$ Logo após o início da radioterapia, citocinas são liberadas do tecido epitelial, como fator de necrose tumoral-alfa (TNF-a), interleucina-1(IL-1) e talvez interleucina-6 (IL-6), iniciando a fase vascular. Na fase vascular ocorre aumento da vascularização subepitelial e liberação de citocinas pró-inflamatórias pelo tecido conjuntivo. ${ }^{43}$ Ocorrem em seqüência danos moleculares irreparáveis na fase $S$ do ciclo celular e morte das células epiteliais basais por apoptose. ${ }^{29,43,44} \mathrm{O}$ tempo do ciclo celular do queratinócito basal é cerca de 4 dias, e como o epitélio bucal apresenta de 3 a 4 camadas, os danos apoptóticos induzidos pela radioterapia na mucosa aparecem aproximadamente 12 dias após o início do tratamento. $^{29}$

$\mathrm{Na}$ fase epitelial, ocorre diminuição, na primeira semana após o início da radioterapia, da proliferação de queratinócitos, induzindo atrofia epitelial. Posteriormente, a partir da segunda semana, há recuperação parcial nas taxas de proliferação levando a repopulação epitelial transiente. ${ }^{24}$ Alguns dados experimentais têm demonstrado aumento da intensidade da mucosite quando os níveis de KGF (fator de crescimento de queratinócitos), importante promotor de proliferação epitelial, se encontram diminuídos, reforçando a idéia de que mecanismos biológicos responsáveis pela repopulação de células epiteliais alteradas também estão afetados pela radiação..$^{45} \mathrm{Em}$ conseqüência da diminuição da proliferação celular, ocorre atrofia persistente do epitélio e exposição do tecido conjuntivo que passa a ser recoberto por camada fibrinopurulenta, rica em neutrófilos. ${ }^{42}$

Quando a dose radioterápica diária é de 200 cGy, as manifestações clínicas da depleção epitelial aparecem mais precocemente do que quando o paciente recebe 180 cGy/dia, devido ao desequilíbrio entre a morte celular e a repopulação epitelial, causado por maior dose diária. ${ }^{46}$ Após a fase ulcerativa, devido provavelmente a eventos de proliferação e diferenciação, o epitélio é restaurado ${ }^{42,43}$ A reparação pode ser retardada se a depleção epitelial celular for intensa e profunda, se houver infiltrado inflamatório significativo e se houver alterações no curso da reparação. ${ }^{46}$

Histopatologicamente, no tecido conjuntivo, a mucosite induzida pela radioterapia não é uma resposta inflamatória aguda ou uma forma agressiva de inflamação crônica. Representa um processo que rememora a fase de reparação da inflamação. ${ }^{27}$ É caracterizado por não demonstrar alterações significativas nas contagens dos linfócitos $\mathrm{T}$ e granulócitos, comparada ao tecido nãoirradiado, e pelo aumento da subpopulação de macrófagos reativos para o anticorpo RM3/1 (macrófagos encontrados em fases tardias de desordens inflamatórias). ${ }^{27,47}$ Os vasos sangüíneos se apresentam com calibres aumentados e há evidência do aumento da permeabilidade vascular e migração de leucócitos ${ }^{22,48}$ (Figura 4). Entre as conseqüências tardias encontradas após a radioterapia em mucosa, observa-se a diminuição no número dos vasos sangüíneos e o aumento no diâmetro do lúmem dos vasos remanescentes, além da predominância de macrófagos RM3/1 positivos. ${ }^{49}$

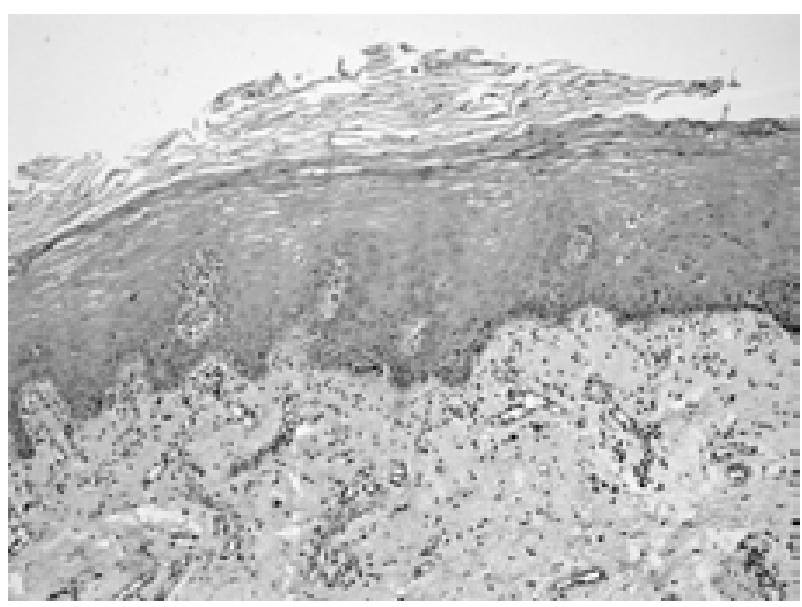

Figura 4. Hiperparaqueratose, camada basal hipercromática, infiltrado inflamatório mononuclear, vasos sangüíneos dilatada em mucosa jugal irradiada em paciente apresentando mucosite grau I (OMS). Hematoxilina e Eosina, 100X.

\section{TRATAMENTO DA MUCOSITE ORAL}

Os tratamentos empregados para a mucosite oral induzida por radioterapia são paliativos e diversificados, envolvem terapêuticas profiláticas e tentativas de atenuar a sintomatologia dolorosa. ${ }^{3,18}$ Embora existam muitos estudos que abordem esse assunto na literatura, poucos foram do tipo duplo-cego ou utilizaram placebos. ${ }^{18}$ As terapias incluem abordagens profiláticas como conscientização para a melhoria na higiene oral, evitar a utilização de alimentos picantes e tabaco, uso de fatores de crescimento (EGF (fator de crescimento epidérmico), GM-CSF (fator estimulante de colônia granulocítico macrofágico, KGF (fator de crescimento para ceratinócitos), sais de alumínio (sucralfato e Maalox®), citocinas (interleucina-11), glutamina citoprotetores e antioxidantes, e sintomáticas como o uso da camomila, 
betametasona, benzidamida, ácido acetilsalicílico, lidocaína, polimixina $\mathrm{E}$, lozenges, tobramicina, laseres de baixa energia e crioterapia, entre outros. ${ }^{3,21,29,38,45,50-61}$ Dentre as terapias mais estudadas e empregadas cita-se o sucralfato, clorexidina, descontaminação seletiva, crioterapia, drogas imunomoduladoras e fatores de crescimento de custos variados. ${ }^{18,21}$ Os trabalhos de pesquisa mais recentes têm apresentado bons resultados com utilização de fatores de crescimento como o GMCSF na prevenção de mucosite oral intensa sugerindo seu futuro uso terapêutico, embora essa tendência não seja unânime. ${ }^{20,23,57,58} \mathrm{Em}$ um estudo em que se administrou GM-CSF em forma de bochecho para 46 pacientes, evidenciou-se redução significativa da severidade da mucosite em 26 pacientes e a conclusão do tratamento com mucosite grau I em 20 pacientes. ${ }^{58}$ Em contrapartida, em um estudo com 35 pacientes submetidos a radioterapia em cabeça e pescoço concomitante a quimioterapia em carcinomas avançados, através de uma análise prospectiva, randomizada, demonstrou que não houve diferenças no grau da mucosite, percepção da dor e infecções cruzadas entre a utilização tópica do GM-CSF e de um bochecho de hidrocortisona. ${ }^{59}$

$\mathrm{O}$ uso da amifostina tem sido indicado por seu potencial citoprotetor em alguns estudos. ${ }^{18}$ Alguns estudos recentes têm demonstrado a eficácia da amifostina intravenosa $(200 \mathrm{mg} / \mathrm{m}(2) / \mathrm{d}) \mathrm{em}$ reduzir toxicidade tecidual pela quimioterapia, xerostomia e na prevenção da mucosite em modelos animais irradiados. ${ }^{60-62}$ Todavia, poucos estudos foram realizados em humanos, freqüentemente não são duplo-cegos e o risco da toxicidade pelo uso da droga ainda são reportados. ${ }^{18,62}$ Outro grande problema referente a utilização das citocinas recombinantes e da amifostina é o custo elevado que faz com que a sua utilização seja inviável para o uso rotineiro. ${ }^{59}$

As tentativas de prevenção e tratamento para a mucosite oral observando as fases de desenvolvimento da mucosite parecem ser inovadoras. ${ }^{63}$ Em harmonia com esse raciocínio, Biron et al ${ }^{53}$ incentivaram a observação das diferentes fases biológicas da mucosite oral para a escolha da terapêutica apropriada. $\mathrm{Na}$ fase vascular, recomenda-se vasoconstrição (crioterapia), citoprotetores e antioxidantes e inibidores de proliferação, como o TGF ß3. Na fase epitelial, devemse utilizar fatores de crescimento ou aceleradores da renovação epitelial, com atuação direta na mucosa, como sucralfato, GM-CSF e KGF. Na fase ulcerativa, bochechos à base de clorexidina em base aquosa e antimicrobianos podem ser indicados. $\mathrm{Na}$ fase de reparação deve-se utilizar laseres de baixa potência. Embora essa sugestão terapêutica seja interessante e completa, não existe consenso no uso das terapêuticas mais comumente indicadas de forma isolada, como mostram estudos antagônicos sobre o uso profilático do sucralfato, na prevenção da mucosite oral ${ }^{22,29,64,65}$ e utilização de antimicrobianos na prevenção ou na redução $\mathrm{da}$ intensidade da mucosite oral. ${ }^{56,36,37} \mathrm{O}$ uso de planejamento radioterápico conformacional têm minorado o desenvolvimento de efeitos colaterais como a xerostomia, como demonstrado em um estudo com 11 pacientes irradiados em cabeça e pescoço, quando da exclusão de uma das parótidas pelo planejamento, apresentaram fluxo salivar muito próximo dos valores antes da radioterapia. ${ }^{6} \mathrm{Um}$ estudo correlacionou a mucosite oral com o planejamento conformacional em 63 pacientes, mostrando bons resultados pela incidência superior de mucosite graus I e II (59\%) sobre mucosite grau III (41\%) e necessidade reduzida de entubação (8\%).?

\section{CONCLUSÃO}

Os aspectos clínicos e a epidemiologia da mucosite oral induzida por radioterapia já estão bem fundamentados. A literatura tem demonstrado o interesse crescente na compreensão dos processos biológicos e na histopatologia da mucosite oral. Técnicas como o planejemento conformacional parecem ser promissoras na redução de efeitos colaterais agudos. $\mathrm{O}$ avanço nas modalidades radioterápicas e na compreensão dos mecanismos biológicos relacionados com a mucosite provavelmente levará a diminuição de sua incidência e ao desenvolvimento de terapias eficazes na prevenção e manejo.

\section{REFERÊNCIAS}

1. Zakrzewska JM. Oral Cancer. BJM. 1999;318(7190):1051-4.

2. Scully C, Porter S. ABC of oral health. Oral cancer. BMJ. 2000;321(7253):97-100.

3. Scully C, Porter S. Oral cancer.West J Med. 2001;174(5):348-51.

4. Chao C, Deasy JO, Markman J, Haynie J, Perez CA, Purdy $\mathrm{JA}$, et al. Functional outcome of parotid gland sparing in patients with head and neck $(\mathrm{H} \& \mathrm{~N})$ cancers receiving intensity-modulated (IMRT) or 3-D radiation therapy. J Radiat Oncol. 2000;48(3 Suppl 1):174.

5. Sannomiya EK, Furukawa S. Different methods of radiotherapy in oral cancer and its complications. Rev Bras Odontol. 2000;57(5):336-8.

6. Claus F, Duthoy W, Boterberg T, De Gersem W, Huys J, Vermeersch $\mathrm{H}$, et al. Intensity modulated radiation therapy for oropharyngeal and oral cavity tumors: clinical use and experience. Oral Oncol. 2002;38(6):597-604.

7. Vineberg KA, Eisbruch A, Coselmon MM, Mcshan DL, Kessler ML, Fraass BA. Is uniform target dose possible in 
IMRT plans in the head and neck? Int J Radiation Oncol Biol Phys. 2002;52(5):1159-72.

8. Pernot M, Luporsi E, Hoffstetter S, Peiffert D, Aletti P, Marchal C, et al. Complications following definitive irradiation for cancers of the oral cavity and the oropharynx (in a series of 1134 patients). Int J Radiat Oncol Biol Phys. 1997;37(3):577-85.

9. Epstein JB, Emerton S, Kolbinson DA, Le ND, Phillips N. Quality of life and oral function following radiotherapy for head and neck cancer. Head Neck.1999;21(1):1-19.

10. Schubert MM, Izutsu KT. Iatrogenic causes of salivary gland dysfunction. J Dent Res. 1987;66(Spec No):680-8.

11. Maxymiw WG, Wood RE. The role of dentistry in head and neck cancer. J Can Dent Assoc. 1989;55(3):193-8.

12. Barrett AW, Porter SR, Scully C, Eveson JW, Griffiths MJ. Oral melanotic macules that develop after radiation therapy. Oral Surg Oral Med Oral Pathol. 1994;77(4):431-4.

13. Garg AK, Malo M. Manifestations and treatment of xerostomia and associated oral effects secondary to head and neck radiation therapy. J Am Dent Assoc. 1997;128(8):1128-33.

14. Morrish RB, Chan E, Silverman S, Meyer J, Fu KK, Greenspan D. Osteoradionecrosis in patients irradiated for head and neck carcinoma. Cancer. 1981;47(8):1980-3.

15. Bonan PR, Pires FR, Lopes MA, di Hipólito O Jr. Evaluation of salivary flow in patients during head and neck radiotherapy. Pesqui Odontol Bras. 2003;17(2):156-60.

16. Spijkervet FK, Van Saene HK, Panders AK, Vermey A, Mehta DM. Scoring irradiation mucositis in head and neck cancer patients. J Oral Pathol Med. 1989;18(3):167-71.

17. Sur RK, Kochhar R, Singh DP. Oral sucralfate in acute radiation oesophagitis. Acta Oncol. 1994;33(1):61-3.

18. Köstler WJ, Hejna M, Wenzel C, Zielinski CC. Oral mucositis complicating chemotherapy and/or radiotherapy: options for prevention and treatment. CA Cancer J Clin. 2001;51(5):290-315.

19. Rovirosa A, Ferre J, Biete A. Granulocyte MacrophageColony-Stimulating factor mouthwashes heal oral ulcers during head and neck radiotherapy. Int J Radiat Oncol Biol Phys. 1998;41(4):747-54.

20. Hejna M, Brodowicz T, Zielinski CC. Local use of GMCSF for severe mucositis. Eur J Cancer. 1999;35 Suppl 3:S14-7.

21. Plevová P. Prevention and treatment of chemotherapy and radiotherapy induced oral mucositis: a review. Oral Oncol. 1999;35(5):453-70.

22. Etiz D, Erkal HS, Serin M, Kucuk B, Hepari A, Elhan AH, et al. Clinical and histopathological evaluation of sucralfate in prevention of oral mucositis induced by radiation therapy in patients with head and neck malignacies. Oral Oncol. 2000;36(1):116-20.

23. Sprinzl GM, Galvan O, de Vries A, Ulmer H, Gunkel AR,
Lukas P, et al. Local application of granulocyte-macrophage colony stimulating factor (GM-CSF) for the treatment of oral mucositis. Eur J Cancer. 2001;37(16):2003-9.

24. Dörr W, Hamilton CS, Boyd T, Reed B, Denham JW. Radiation-induced changes in cellularity and proliferation in human oral mucosa. Int J Radiat Oncol Biol Phys. 2001;52(4):911-7.

25. Leung WK, Dassanayake RS, Yau JY, Jin LJ, Yam WC, Samaranayake LP. Oral colonization, phenotypic, and genotypic profiles of Candida species in irradiated, dentate, xerostomic nasopharyngeal carcinoma survivors. J Clin Microbiol. 2000;38(6):2219-26.

26. Trotti A, Bellm LA, Epstein JB, Frame D, Fuchs HJ, Gwede $\mathrm{CK}$, et al. Mucositis incidence, severity and associated outcomes in patients with head and neck cancer receiving radiotherapy with or without chemotherapy: a systematic literature review. Radiother Oncol. 2003;66(3):253-62.

27. Handschel J, Sunderkötter C, Prott FJ, Meyer U, KruseLösler B, Joos U. Increase of RM 3/1- positive macrophages in radiation induced oral mucositis. J Pathol. 2001;193(2):242-7.

28. Hlavaty TE, Fisher R, Robbins MC, Wheeler J, Funk G, Hussey $\mathrm{DH}$. Radiotherapy for head and neck maligancies is associated with increased salivary platelet- activating factor content. Int J Radiat Oncol Biol Phys. 1996;36(1 Suppl 1):S236.

29. Scully C, Epstein JB. Oral health for the cancer patient. Oral Oncol Eur J Cancer. 1996;32B:281-92.

30. Denham JW, Peters LJ, Johansen J, Poulsen M, Lamb DS, Hindley A, et al. Do acute mucosal reactions lead to consequential late reactions in patients with head and neck cancer? Radiother Oncol. 1999;52(2):57-64.

31. Dumbrigue HB, Sandow PL, Nguyen KH, HumphreysBeher MG. Salivary epidermal growth factor levels decrease in patients receiving radiation therapy to the head and neck. Oral Surg Oral Med Oral Pathol Oral Radiol Endod. 2000;89(6):710-6.

32. Epstein JB, Gorsky M, Guglietta A, Le N, Sonis ST. The correlation between epidermal growth factor levels in saliva and the severity of oral mucositis during oropharyngeal radiation therapy. Cancer. 2000;89(11):2258-65.

33. Bentzen SM, Saunders MI, Dische S, Bond SJ. Radiotherapy-related early morbidity in head and neck cancer: quantitative clinical radiobiology as deduced from the CHART trial. Radiother Oncol. 2001;60(2):123-35.

34. Paula CR, Sampaio MC, Birman EG, Siqueira AM. Oral yeasts in patients with cancer of the mouth, before and during radiotherapy. Micopathologia. 1990;112(2):119-24.

35. Ramirez-Amador V, Silverman S, Mayer P, Tyler M, Quivey J. Candidal colonization and oral candidiasis in patients undergoing oral and pharyngeal radiation therapy. Oral Surg Oral Med Oral Pathol Oral Radiol Endod. 1997;84(2):149-53. 
36. Wijers OB, Levendag PC, Harms ER, Gan-Teng AM, Schmitz PI, Hendriks WD, et al. Mucositis reduction by selective elimination of oral flora in irradited cancers of head and neck: a placebo-controlled double-blind randomized study. Int J Radiat Oncol Biol Phys. 2001;50(2):343-52.

37. Stokman MA, Spijkervet FK, Burlage FR, Dijkstra PU, Manson WL, de Vries EG, et al. Oral mucositis and selective elimination of oral flora in head and neck cancer patients receiving radiotherapy: a double-blind randomized clinical trial. Br J Cancer. 2003;88(7):1012-6.

38. Nicolatou-Galitis O, Sotiropoulou-Lontou A, Velegraki A, Pissakas G, Kolitsi G, Kyprianou K, et al. Oral candidiasis in head and neck cancer patients receiving radiotherapy with amifostine cytoprotection. Oral Oncol. 2003;39(4):397-401.

39. Blozis GG, Robinson JE. Oral tissue changes caused by radiation therapy and their management. Dent Clin North Am. 1968;643-56.

40. Rothwell BR. Prevention and treatment of the orofacial complications of radiotherapy. J Am Dent Assoc. 1987;114(3):316-22.

41. Stokman MA, Spijkervet FK, Wymenga AN, Burlage FR, Timens W, Roodenburg JL, et al. Quantification of oral mucositis due to radiotherapy by determining viability and maturation of epithelial cells. J Oral Pathol Med. 2002;31(3):153-7.

42. Pico JL, Avila-Garavito A, Naccache P. Mucositis: its occurence, consequences, and treatment in the oncology setting. Oncologist. 1998;3(6):446-51.

43. Sonis ST. Mucositis as a biological process: a new hypotesis for the development of chemotherapy-induced stomatotoxicity. Oral Oncol. 1998;34(1):39-43.

44. Sonis ST. Oral complications. 4th ed. In: Holland JF, Frei E, et al. Cancer medicine. Philadelphia: Williams \& Wilkins;1997. p. 3255-64.

45. Dörr W, Spekl K, Farrell CL. Amelioration of acute oral mucositis by keratinocyte growth factor: fractioned irradiation. Int J Radiat Oncol Biol Phys. 2002;54(1):245-51.

46. Denham JW, Hauer-Jensen M. The radiotherapeutic injury: a complex wound. Radiother Oncol. 2002;63(2):129-45.

47. Zwadlo G, Voegeli R, Osthoff KS, Sorg C. A monoclonal antibody to a novel differentiation antigen on human macrophages associated with the down-regulatory phase of the inflammatory process. Exp Cell Biol. 1987;55(6):295-7.

48. Handschel J, Prott FJ, Sunderkotter C, Metze D, Meyer U, Joos U. Irradiation induces increase of adhesion molecules and accumulation of beta2-integrin-expressing cell in humans. Int J Radiat Oncol Biol Phys. 1999;45(2):475-81.

49. Handschel J, Sunderkotter C, Kruse-Losler B, Prott FJ, Meyer U, Piffko J, et al. Late effects of radiotherapy on oral mucosa in humans. Eur J Oral Sci. 2001;109(2):95-102.

50. Wright WE, Haller JM, Harlow SA, Pizzo PA. An oral disease prevention program for patients receiving radiation and chemotherapy. J Am Dent Assoc. 1985;110(1):43-7.

51. Sonis ST, Costa JW Jr, Evitts SM, Lindquist LE, Nicolson M. Epidermal growth factor on ulcerative mucositis in hamsters that receive cancer chemotherapy. Oral Surg Oral Med Oral Pathol. 1992;74(6):749-55.

52. Cowen D, Tardieu C, Schubert M, Peterson D, Resbeut $\mathrm{M}$, Faucher $\mathrm{C}$, et al. Low energy helium-neon laser in the prevention of oral mucositis in patients undergoing bone marrow transplant: results of a double blind randomized trial. Int J Radiat Oncol Biol Phys. 1997;38(4):697-703.

53. Biron P, Sebban C, Gourmet R, Chvetzoff G, Philip I, Blay JY. Research controversies in managment of oral mucositis. Support Care Cancer. 2000;8(1):68-71.

54. Sonis ST, Peterson RL, Edwards LJ, Lucey CA, Wang L, Mason L, et al. Defining mechanisms of action of interleukin-11 on the progression of radiation-induced oral mucositis in hamsters. Oral Oncol. 2000;36(4):373-81.

55. Huang EY, Leung SW, Wang CJ, Chen HC, Sun LM, Fang FM, et al. Oral glutamine to alleviate radiation-induced oral mucositis: a pilot randomized trial. Int J Radiat Oncol Biol Phys. 2000;46(3):535-9.

56. Adamietz IA, Rahn R, Böttcher HD, SchäferV, Reimer K, Fleischer W. Povidone-iodine to prevent mucositis in patients during antineoplastic radiochemoterapy. Dermatology. 1997;195 Suppl:57-61.

57. Makkonen TA, Minn H, Jekunen A, Vilja P, Tuominen J, Joensuu H. Granulocyte macrophage-colony stimulating factor (GM-CSF) and sucralfate in prevention of radiationinduced mucositis: a prospective randomized study. Int J Radiat Oncol Biol Phys. 2000;46(3):525-34.

58. Nicolatou-Galitis O, Dardoufas K, Markoulatos P, Sotiropoulou-Lontou A, Kyprianou K, Kolitsi G, et al. Oral pseudomembranous candidiasis, herpes simplex virus-1 infection, and oral mucositis in head and neck cancer patients receiving radiotherapy and granulocytemacrophage colony-stimulating factor (GM-CSF) mouthwash. J Oral Pathol Med. 2001;30(8):471-80.

59. Sprinzl GM, Galvan O, de Vries A, Ulmer H, Gunkel AR, Lukas P, et al. Local application of granulocyte-macrophage colony stimulating factor (GM-CSF) for the treatment of oral mucositis. Eur J Cancer. 2001;37(16):2003-9.

60. Suntharalingam M, Jaboin J, Taylor R, WolfJ, Banglore M, Van Echo D, etal. The evaluation of amifostine for mucosal protection in patients with advanced loco-regional squamous cell carcinomas of the head and neck (SCCHN) treated with concurrent weekly carboplatin, paclitaxel, and daily radiotherapy (RT). Semin Oncol. 2004;31(6 Suppl 18):S2-7.

61. Cassatt DR, Fazenbaker CA, Bachy CM, Kifle G, McCarthy MP. Amifostine (ETHYOL) protects rats from mucositis resulting from fractionated or hyperfractionated radiation exposure. Int J Radiat Oncol Biol Phys. 2005;61(3):901-7. 
62. Bourhis J, Rosine D. Radioprotective effect of amifostine in patients with head and neck squamous cell carcinoma. Semin Oncol. 2002;29(6 Suppl 19):61-2.

63. Sutherland SE, Browman GP. Prophylaxis of oral mucositis in irradiated head and neck cancer patients: a proposed classification scheme of interventions and meta-analysis of randomized controlled trials. Int J Radiat Oncol Biol Phys. 2001;49(4):917-30.

64. Lievens Y, Haustermans K, Van der Weyngaert D, Van den Bogaert W, Scalliet P, Hutsebaut L, et al. Does sucralfate reduced the acute side-effects in head and neck cancer treated with radiotherapy? A double-blind randomized trial.
Radiother Oncol. 1998;47(2):149-53.

65. Saarilahti K, Kajanti M, Joensuu T, Kouri M, Joensuu H. Comparison of granulocyte-macrophage colonystimulating factor and sucralfate mouthwashes in the prevention of radiation-induced mucositis: a double-blind prospective randomized phase III study. Int J Radiat Oncol Biol Phys. 2002;54(2):479-85.

66. Kam MK, Teo PM, Chau RM, Cheung KY, Choi PH, Kwan WH, et al. Treatment of nasopharyngeal carcinoma with intensity-modulated radiotherapy: the Hong Kong experience. Int J Radiat Oncol Biol Phys. 2004;60(5):1440-50. 